\title{
Rigomer Bazin et la Restauration : penser la république dans la monarchie
}

\section{Pierre Serna}

\section{(2) OpenEdition \\ 1 Journals}

\section{Édition électronique}

URL : https://journals.openedition.org/ahrf/442

DOI : 10.4000/ahrf.442

ISSN : 1952-403X

Éditeur :

Armand Colin, Société des études robespierristes

\section{Édition imprimée}

Date de publication : 1 septembre 2001

Pagination : 53-76

ISSN : 0003-4436

\section{Référence électronique}

Pierre Serna, «Rigomer Bazin et la Restauration : penser la république dans la monarchie », Annales historiques de la Révolution française [En ligne], 325 | juillet-septembre 2001, mis en ligne le 10 avril 2006, consulté le 24 avril 2022. URL : http://journals.openedition.org/ahrf/442 ; DOI : https://doi.org/ 10.4000/ahrf.442

Ce document a été généré automatiquement le 24 avril 2022.

Tous droits réservés 


\title{
Rigomer Bazin et la Restauration : penser la république dans la monarchie
}

\author{
Pierre Serna
}

1 Les chantiers de recherches ouverts par le bicentenaire ont renouvelé les domaines des pratiques et des idées politiques ${ }^{1}$. Ainsi, l'étude de « la dévolution et la répartition de l'autorité et du pouvoir au sein d'un groupe humain donné, et l'étude des tensions, des antagonismes et des conflits en découlant $\|^{2}$, s'est enrichi d'une histoire des gestes de la pratique militante (vote, réunion, manifestations) ainsi que de l'étude des représentations (symbole, lecture, programme artistique, langue) ${ }^{3}$. De plus, la dimension temporelle, désormais prise en compte de façon à construire une histoire des personnes en Révolution plutôt que l'histoire d'un événement traversé par des biographies, a permis d'interroger le passé de la Révolution'. L'individuation de la sphère de l'opinion publique, par exemple, et la naissance d'une culture de la contestation mise en valeur par Keith Baker, ont souligné les tensions apparues dans le débat public des dernières décennies de la monarchie. En revanche, la prosopographie des Constituants proposée par T.Tackett a donné à comprendre combien la radicalité des débats, dès les premières semaines des États généraux, s'expliquait par l'origine de ces députés dont l'étude précise renversait toutes les idées convenues sur la sociologie des 1200 représentants du royaume.

2 Fort de ces acquis, il s'agit ici de poser l'hypothèse du retournement de la perspective chronologique de la recherche, afin d'aborder le devenir de tous ceux qui s'étaient engagés dans la Révolution à des titres divers et qui avaient, selon le mot de Sieyès, « survécu... » Ce qui était pertinent pour mieux comprendre 1789, l'était-il pour saisir 1799, ou 1815 ? Quelle part la culture et l'expérience politique de la Révolution allaientelles jouer dans le destin de tous ceux qui, engagés radicalement dans le processus révolutionnaire, ne pouvaient que se définir comme des opposants à certains aspects de la Constitution directoriale et, plus particulièrement encore, au Consulat, à l'Empire et à la Restauration. 
1799-1815 : les républicains démocrates ont survécu, eux aussi

3 Partir à la recherche de ce groupe de "Républicains-démocrates », tels qu'Alphonse Aulard les avait baptisés, fortement marqué par l'expérience terroriste assumée et, cependant, loin de représenter la mouvance figée d'un robespierrisme nostalgique, revient à approfondir les réseaux républicains de la gauche directoriale et ses revendications d'un régime de démocratie représentative ${ }^{5}$. Parmi ces derniers, un homme jeune, RigomerBazin, trente ans en l'anVIII, va développer une pensée originale et la soutenir jusqu'en 1818... lorsqu'il n'est pas en prison pour la radicalité de ses prises de position, ou surveillé, comme il se doit, par toutes les polices de tous les régimes, de 1795 à 1818 . Placé du côté de l'aile gauche la plus radicale du mouvement démocratique, il propose, en 1815, contre toute attente, pour la Charte octroyée par Louis XVIII, une lecture se résumant à l'opportunité d'établir un régime constitutionnel fondé sur la représentation!

4 Du Directoire à la Restauration... Ces deux régimes, parents pauvres del'historiographie, constituent pourtant des moments de profonde réflexion politique qui, paradoxalement, permettent à certains démocrates, oubliés ou réduits au silence, de se manifester et de proposer, loin de les attaquer seulement, des solutions d'aménagements des deux textes de la Constitution de l'AnIII et de la Charte, d'un point de vue démocratique pour le Directoire, sous l'angle républicain pour la Restauration. Il reste encore à étudier l'inventaire des documents qui proposent une façon républicaine de lire la Charte constitutionnelle ${ }^{6}$. Pierre Rosanvallon a individualisé deux écritures opposées de l'histoire de cette période qui se voit écartelée entre une lecture jacobine et celle d'un "moment anglais de l'Histoire de France", perceptible dans les deux Chartes de 1815 et $1830^{7}$, développant une extension des libertés et des progrès du gouvernement représentatif. La Charte constitutionnelle offrait, en effet, de nombreuses interprétations. En 1817, Bazin a pu, et il n'est pas le seul, imaginer faute de mieux et étant donné les circonstances, une "République royale ", une " Monarchie représentative $~^{8}$ capable de réunir les antagonismes des Français, de même qu'il a pu croire le moment venu d'inventer un régime, capable d'incarner les deux formules politiques qui jusque-là avaient engendré une lutte fratricide et qui pouvaient désormais être au fondement de la régénération du pays. C'était le rêve d'un élan ni patriotique, ni dynastique, mais d'un « moment américain " pour la France, celui d'une république avec, à sa tête, un chef de l'exécutif doté de prérogatives importantes... expérience finalement ratée 9 .

Bazin, entre transparence et secrets

5 Rigomer Bazin donc! Il est un journaliste démocrate et un écrivain constitutionnel. Avec d'autres, il offre un parcours biographique illustrant la génération des démocrates, nés entre 1760-1770, et leurs vicissitudes, à travers tous les régimes qui les proscrivent, de la Terreur à la Restauration, cultivant ce qu'ils appellent «leur invariabilité » face à toutes formes de proscription ou d'exclusion, revendiquant le privilège d'une fidélité indéfectible à un idéal, en ces temps de recomposition permanente des alliances et des allégeances politiques ${ }^{10}$. Né en 1769 , Rigomer Bazin, fils et petit-fils d'épiciers manceaux, débute une carrière classique de révolutionnaire dans la Garde nationale. Membre du comité de défense républicaine contre les Vendéens en 1793, il se retrouve devant le tribunal révolutionnaire pour s'être opposé au représentant en mission Garnier de Saintes. Acquitté, il est enfermé, puis libéré au 9 Thermidor ${ }^{11}$. La période qui suit va le voir développer son talent de journaliste politique, tour à tour au Mans, où il 
occupe le poste de rédacteur de La Chronique de la Sarthe, et à Paris, où il devient, en l'anVII, un militant, au Club du Manège. Il est surtout l'âme du journal Le Démocrate (12), qui forme avec le Journal des Hommes libres, une des deux tribunes où la réflexion politique propose une lecture démocratique de la Constitution de l'anIII ${ }^{13}$.

Parmi d'autres idées, Bazin développe la nécessité de constituer en régime d'assemblée, une opposition politique, seul contrepoids aux gouvernants, devenu nécessaire. Il défend l'impératif d'une construction de partis forts et capables de résister constitutionnellement aux gouvernements, par le jeu d'élections devant contribuer à l'alternance des hommes au pouvoir ${ }^{14}$. La vitalité du corps civique s'édifie sur la capacité de ceux qui gouvernent à accepter, à protéger même, ceux qui contestent leur manière de conduire les affaires ${ }^{15}$. Seuls les droits de la minorité, parfois confondus avec le "peuple » définissent la valeur du système politique en entier. Les élections fréquentes matérialisent la souveraineté populaire, ainsi capable d'exercer un réel contrôle sur les représentants, sans les brider dans leur fonction législative. Sous le Consulat et l'Empire, il développe cette idée malgré la censure politique de plus en plus contraignante.

7 En 1808, il participe à la conspiration du général Malet. Longuement interrogé par la police, Bazin tente de se protéger en gardant le silence, ou en répondant de façon évasive, lorsqu'il est contraint de reconnaître l'existence de certains liens avec des personnes compromises ${ }^{16}$. Sa fidélité au secret partagé lui vaut de terminer l'Empire dans les geôles où il demeure six ans et réalise, de son propre aveu, des rencontres fructueuses avec des proscrits royalistes ${ }^{17}$. Un an auparavant, en 1807, Bazin avait attiré l'attention de la police par une publication: Les Lettres philosophiques, de parution régulière, qui se donne pour but "d'ouvrir entre toutes les personnes éclairées une correspondance publique, où les opinions diverses sont soumises à un libre examen ${ }^{18}$. Dans un contexte qui voit les libertés politiques davantage réduites, par la suppression du Tribunat, ou par l'interdit fait aux rédacteurs de La Décade de ne plus faire paraître leur journal, l'initiative de Bazin ne manque pas de faire sens et signifie un acte de résistance politique notoire. Dans la mesure où la police impériale veille de façon efficace et où l'autocensure est également perceptible, le texte livre autant d'indices qu'il le peut. Ainsi, l'auteur s'attache d'abord à préciser qu'» aujourd'hui, le mérite a droit aux emplois... voilà la vraie liberté. Partout où de tels principes sont proclamés par le législateur, il y a des citoyens : quelle que soit la forme du gouvernement, il est libéral ». Cependant, les censeurs veillent... d'autant que les propositions politiques deviennent, au fil des parutions, toujours plus subversives. La loi, constate Bazin, en tant que lieu d'élaboration du lien entre éthique et politique doit permettre à tous, dans un régime libéral de concilier les contradictions inhérentes à la communauté qui se trouve divisée entre l'instinct individualiste de chacun et la nécessité théorique de sacrifier l'individu au salut du groupe. La loi, garantie par la Constitution libérale, permet de rejouer en permanence les exigences de cet entre-deux, les désirs de l'individu et les exigences de la communauté, et peut être considérée comme l'instrument de la conservation d'un régime de libertés, de toutes les libertés, « réunion des vertus publiques et privées $»^{19}$.

8 Il est difficile d'aller beaucoup plus loin en 1807. D'ailleurs, c'en est trop déjà... et la police interdit une parution qu'elle soupçonne, vraisemblablement avec raison, de divulguer des informations codées à un réseau de lecteurs sûrement alertés par la signature de cette lettre: Philadelphe, soit le nom, connu des services d'espions de 
l'empereur, d'un mystérieux réseau composé surtout de militaires et de démocrates, partageant un idéal maçonnique et qui voudrait attenter à la vie de «l'usurpateur $»^{20}$. En tout cas, la liste compromettante des abonnés des Lettres philosophiques, montre toute une microsociété encore animée d'un farouche esprit anti-napoléonien ${ }^{21}$. Dès lors, la police ne cesse de traquer Bazin jusqu'à ce qu'il se retrouve en geôle, pour de longues années. Il ne recouvre sa liberté qu'en 1814, date à laquelle, sitôt sorti de prison, il poursuit son travail de journaliste d'opposition. C'est, après 1799 et 1807, le troisième acte de l'approche de ce personnage. La période qui s'ouvre en 1814 et s'achève en 1818 est pour lui riche du point de vue de la réflexion politique : elle consiste en une défense du principe de la monarchie constitutionnelle - ce qui a pu sembler paradoxal à certains - tout en confirmant par ailleurs, par ses critiques sur l'actualité politique, sa figure d'opposant irréductible.

La Restauration : la liberté de parole retrouvée

Dès 1814 donc, Bazin, inlassablement, reprend sa plume. « Après six ans de captivité, je renais à la lumière.» Il évoque le retour aux «sages conceptions de l'Assemblée constituante sanctionnées par Louis XVI" ${ }^{22}$. Les Lettres philosophiques peuvent reparaitre, suivies d'une abondante littérature politique qui va prendre la forme de deux volumes en $1815^{23}$ et en 1817, paraissant tous deux sous le même titre Le Lynx, la métaphore de la bête politique et lucide. C'est le second volume qui est étudié ici : il est le dernier écrit d'importance du journaliste ; il est le témoignage le plus tardif laissé par le Sarthois sur la situation de la Restauration; il est, enfin, le lieu où s'élabore de la façon la plus argumentée, l'interprétation de la Charte. En un peu plus de plus de quatre cents pages, l'auteur présente une lecture originale du retour des Bourbons. Loin de s'opposer à LouisXVIII et au principe monarchique qu'il incarne, loin de rejeter sans discussion le système né de la Restauration, l'irréductible républicain-démocrate propose une lecture résolument positive et paradoxale de la Charte et développe l'ensemble des éléments qui rendraient possible l'expérience d'une monarchie constitutionnelle ${ }^{24}$. Au-delà du cas Bazin, ce qui est interrogé, dans la poursuite du processus engagé sous le Directoire, c'est la modernité d'un jeu politique initiant l'histoire du parlementarisme en France, et le réveil de tous ces républicains dont Madame de Staël elle-même avoue qu'ils avaient « retrouvé leur ancienne vigueur " 25 .

À quel type de représentations obéit Bazin ? Sur quels espoirs se fonde-t-il pour parier quant aux chances constitutionnelles qu'avait ce gouvernement de reconstruire le tissu politique à partir des garanties exprimées dans la Charte ? Quelles étaient les capacités des anciens révolutionnaires à faire entendre leur voix et à faire partager leur point de vue ? Comment pouvaient-ils justifier la défense d'un régime honni quelques années auparavant? Comment comptaient-ils reconstruire la souveraineté populaire, base de tout système républicain, par le haut, c'est-à-dire par une Charte et, qui plus est, octroyée par un roi $^{26}$ ?...

11 L'ensemble des brochures réunies par Bazin, parce que celles-ci, dans le fond, sont demeurées républicaines, répond de façon originale, aux questions dont dépend le sort de la Restauration, de sa légitimité et de sa survie: soit le maintien des principes d'égalité et de liberté avec les règles de la monarchie bourbonienne ${ }^{27}$. À la lecture, elles peuvent donner l'impression d'une série de textes mis bout à bout sans grande cohérence, l'un abordant l'éducation, l'autre un projet d'assurance sociale, un autre le fonctionnement de la justice. En réalité ces textes offrent une représentation complexe d'un système politique inachevé en tant que tel, où différentes facettes d'un projet de 
société présenté, doivent être améliorées, à l'aune d'un horizon démocratique sans cesse repoussé. Il n'est pas inutile de rappeler les conditions de la genèse mouvementée de ces pages et de leur publication délicate. En effet, du 21 septembre 1816 au 25 juin 1817, entre le préfet de la Sarthe et le ministère de la Police générale, une correspondance est échangée, à propos "d'un des athlètes les plus fougueux de la Révolution $»^{28}$. Et Bazin, lui-même, non content de publier de nombreux petits ouvrages, dans lesquels le préfet voudrait « comprimer impitoyablement toute saillie révolutionnaire $»^{29}$, dépose une plainte contre le premier fonctionnaire du département ${ }^{30}$. Le plaignant justifie sa démarche par le fait que le préfet a voulu exercer un droit de censure sur un texte intitulé Les Lettres constitutionnelles, alors que la loi du 21 octobre 1815 sur le contrôle des parutions n'accordait pas au fonctionnaire zélé le droit d'interdire de façon préventive les écrits de moins de vingt pages - c'est là, à n'en pas douter la raison qui explique la concision et la multiplication des parutions chez Bazin -. Pour le représentant de l'ordre, cette interdiction est légitimée par le fait qu'il importe que "des écrivains tels que Bazin, qui s'est fait connaître pendant l'interrègne d'une manière très défavorable, ne puissent agiter les esprits, surtout dans les départements, et troubler la tranquillité publique $»^{31}$. Il est vrai que les brochures sont tirées à mille ou deux mille exemplaires et bénéficient d'un succès non négligeable auprès d'un lectorat resté fidèle à l'idée républicaine. Une quinzaine de dépositaires non professionnels, mais aussi des libraires, à Angers, à Laval, à Rouen, sont autant de relais pour les idées de Bazin, subversives aux regards des autorités ${ }^{32}$.

$\mathrm{Au}$ mois de novembre 1816, le préfet informe le ministre qu'il a fait saisir toute une édition du Catéchisme politique suivi de Tout est Bien ${ }^{33}$. L'ouvrage de Bazin est traité de " catéchisme à l'égard des factieux ", d'autant que la deuxième partie suggère qu'un roi libre n'aurait pas donné une Charte, mais une Constitution. Enfin le 31 décembre 1816, le tribunal civil et correctionnel du Mans, saisi de l'affaire, condamne l'auteur démocrate à six mois d'emprisonnement, à cinquante francs d'amende et à cinq ans de surveillance par la haute police d'État, pour écrits inconvenants ${ }^{34}$. Cependant, Bazin parvient à faire appel auprès de la cour d'Angers qui, loin de confirmer le précédent jugement, absout le requérant, le 1er février 1817, et ce malgré le réquisitoire fort sévère de l'avocat général qui traite au passage le journaliste de "folliculaire, pamphlétaire, et écrivain populacier $\aleph^{35}$. La reconnaissance - in fine - du bien fondé de la requête est la preuve qu'une juridiction compétente peut demeurer soucieuse, en 1817, de la liberté d'expression des individus et qu'elle se montre capable de donner à la justice un visage plus libéral. Le signe politique émis a dû paraître assez fort à Bazin pour qu'il ose, peu après, publier l'ensemble de ses brochures, sous la forme d'un livre intitulé Le Lynx. L'auteur salue la relative clémence dont il a été l'objet et l'attribue à un nouveau climat politique... «Les électeurs représenteront enfin, dans toute leur étendue, l'industrie et la propriété... et la responsabilité légale des ministres deviendra bientôt une garantie réelle pour la nation et pour eux-mêmes... Que d'encouragements pour l'écrivain constitutionnel! $»^{36}$, qui rappelle l'article VIII de la Charte: "Les Français ont le droit de publier et de faire imprimer leurs opinions, en se conformant aux lois qui doivent réprimer les abus de cette liberté ${ }^{37}$.

Défendre le système représentatif

Dès le début de l'ouvrage, Bazin souligne la singularité de la Restauration. S'adressant à Chateaubriand, devenu depuis l'Empire un interlocuteur emblématique, l'auteur écrit : "Vous vous écriez: où sommes-nous, où allons-nous? Et moi je vous demande où vous êtes, où vous prétendez aller ${ }^{38}$. Pour Bazin, la réponse s'impose $:$ il s'agit d'un acte de 
la part du souverain qui garantisse les droits des individus et de la collectivité nationale... « Le système représentatif est sur le point de s'établir presque partout ; et écrit-il, en 1814 - la France a retenti des mots Constitution libérale, prononcés par son roi $»^{39}$. Dans un autre écrit, il a soin d'attaquer l'attitude de ceux qu'ils surnomment «les féodaux ", parce qu'ils n'acceptent pas «le système libéral et constitutionnel » ${ }^{40}$. La défense de la Charte, dans sa valeur constitutionnelle, se transforme en leitmotiv, tout au long des pages. Le texte du 4juin 1814 devient la référence sur laquelle s'articule toute l'argumentation du républicain qui ne peut se déclarer. En 1816, dans Le Catéchisme politique à l'égard des Constitutionnels, il se consacre à la description des nouvelles conditions institutionnelles nées de la proclamation de $1814^{41}$.

«Demande : Qu'est-ce qu'un roi?

Réponse : C'est le chef d'un Peuple libre. Il a fait une Charte pour donner des bornes à sa propre puissance, pour consacrer l'égalité des Français devant la loi, et les droits qui en dérivent; pour leur recommander l'oubli du passé, pour que les anciennes inimitiés cessent; et pour que la concorde, assise à côté de lui sur le trône, règne sur nos cœurs, gouverne nos sentiments et nos actions $»^{42}$.

Certes, le mot Charte a remplacé la Constitution. Bazin explique d'ailleurs dans une note, que c'est un terme féodal mais qui, cependant, selon l'usage fait en Angleterre, signifie les concessions faites par des rois à la nation entière. Dans un autre texte, prudent, il a soin d'appuyer sa démonstration sur le parcours biographique du roi qu'il associe à l'histoire de la pré-révolution : « Louis XVIII s'est expatrié pour fuir de grands dangers. Il n'en avait pas moins acquiescé deux ans auparavant, au principe fondamental de la Révolution, je veux dire à l'égalité des droits... La Charte contient l'expression de ses sentiments: c'est sa volonté écrite $»^{43}$. En s'en tenant de façon stricte à la Charte, Bazin a bien conscience de camper sur une position qui, loin d'être un pis-aller, est bien la défense de l'héritage révolutionnaire. La suite présente un dialogue entre Pierre, un paysan, et Ariste, un propriétaire, résumé à un commentaire libéral de La Charte.

De la Charte à la loi, le passage se fait avec hardiesse. Poursuivant sa réflexion dans Le Catéchisme constitutionnel, Bazin, par glissement sémantique, parvient à évoquer la loi, dont l'importance est égale à l'acte constitutionnel :

« Demande : Qu'est-ce que la loi ?

Réponse: C'est un acte délibéré dans les deux Chambres, sanctionné par le roi, transmis par les ministres aux agents préposés à son exécution...

Demande : Quelle est la garantie du citoyen contre la violation de la loi ?

Réponse : La garantie du citoyen est premièrement, dans le droit de remontrance envers le fonctionnaire qui a violé la loi ; secondement, dans le droit de réclamation envers le ministre que cela concerne; troisièmement, dans le recours aux tribunaux ; quatrièmement, dans l'adresse au roi ; cinquièmement, dans le droit de pétition aux deux Chambres ; sixièmement, dans l'opinion publique ${ }^{44}$.

À ce titre, il est révélateur, dans la disposition même des recours, que le roi n'intervenant pas comme ultime possibilité, voie sa souveraineté limitée, et par le droit de pétition, émanation des citoyens regroupés selon leur opinion politique, et par l'opinion publique, comprise comme la nation dans son intégralité : c'est-à-dire non seulement les représentants et le corps électoral, mais aussi l'ensemble des Français dont les revenus ne permettent pas encore une participation aux élections ${ }^{45}$. Finalement, le publiciste a soin d'inscrire son propos dans une tradition historique. En promulguant la Charte constitutionnelle, Louis XVIII n'a fait que poursuivre une coutume ancienne: "Le régime constitutionnel et représentatif fut celui de nos 
ancêtres", comme le prouvent les anciens Champs de Mars. "C'est le système représentatif qui nous sauvera: la monarchie sans la Charte serait un abyme dans lequel s'engloutiraient le trône, l'autel et la patrie $»^{46}$. Pour que la "monarchie représentative" fonctionne désormais, il faut que dans l'espace politique soient garantis, la liberté de la presse, la maturité de l'opinion publique, l'existence des partis, les droits de l'opposition, l'élargissement possible du corps électoral. Or l'auteur de 1816 , qui, en 1817, republie le texte à l'identique, a également conscience que les règles $\mathrm{du}$ jeu politique, non seulement ne jouent plus en faveur d'une lecture constitutionnelle de la Restauration, mais que les libertés, longtemps espérées, un temps aperçues, se voient menacées, et dans l'entourage du roi et encore dans les deux Chambres. Aussi a-t-il soin de rappeler avec insistance qu'il n'y a point de " gouvernement représentatif sans la liberté de la presse " $^{47}$. À la fin de l'ouvrage, après avoir évoqué les discussions houleuses de la session de 1816 et le courage des anciens vétérans, mais aussi celui d'un membre de l'ancienne noblesse, le duc de Broglie qui, à la séance du 6 février 1817, a défendu de façon généreuse et patriote un projet de loi relatif à la liberté individuelle, Bazin réclame une union des Français dans la jouissance de leurs droits, un respect des publications par la proclamation de la liberté de la presse, liée à son tour à la liberté des individus, jouissant d'un droit de réunion ${ }^{48}$.

Les partis politiques et l'opinion publique dans la France de 1815

À plusieurs reprises, Bazin revient sur la description des forces politiques, regroupées, parfois arbitrairement, sous la dénomination de partis, désignés tour à tour de manière différente. De façon quelque peu réductrice, il distingue «les défenseurs de la vieille souche des abus que le siècle a déracinée et qui appellent à leur aide les théories oppressives, et en face, " les rejetons vigoureux destinés à reproduire le peuple franc, et qui soutiennent que la France est rendue au régime représentatif $»^{49}$. Plus loin, la remarque s'affine et l'auteur tente même une quantification des forces, divisées en trois familles qui vont désormais structurer le champ politique du xixesiècle avec plus ou moins de bonheur. D'abord, les féodaux, qui ne regrouperaient que la petite Église, celle qui a refusé le Concordat, cinq à six cents paysans mystifiés par la clandestinité, une partie des émigrés rentrés en 1814, quelques anciens nobles, de riches bourgeois plus ennemis de la Révolution que de la liberté; en tout, un million de personnes. Ensuite, les neutres, ceux qui sont perçus du côté du bon sens et du repos civil, mais que Bazin renvoie aux pages sanglantes du Moniteur de 1794, lorsqu'ils accompagnaient tous les partis à l'échafaud, toujours prompts à se mettre du côté du plus fort; en tout, 6 millions de personnes. Enfin, les constitutionnels, c'est-à-dire le roi, les princes, les ministres, la Chambre des pairs, la Chambre des députés, les représentants de l'administration, le clergé concordataire, les corps savants et littéraires, la vieille armée et l'actuelle, les 9/10e des campagnes, les 9/10e des villes, les patriotes dans toutes leurs variétés, et les 15 millions d'intéressés par la question des biens nationaux; pas moins de 20millions de personnes, selon Bazin, d'un optimisme... calculé.

Si le calcul était aussi juste que la prévision optimiste de Bazin le laisse envisager, les inquiétudes n'auraient pas lieu d'être. Or, il apparaît, à la lecture, que c'est bien la composition de ce troisième parti, tellement hétéroclite qu'à son tour, il en devient introuvable, en 1816, qui explique la fragilité du compromis politique de ces premières années de fonctionnement de la monarchie restaurée. La fin de l'œuvre, contemporaine du début de l'année 1817 et de ces grands débats sur l'armée et les élections, présente un chapitre consacré aux problèmes Des Partis en France. Si les clivages n'ont 
fondamentalement pas changé entre les trois forces politiques, la qualité de dénomination s'est améliorée et, avec elle, la claire perception des programmes qui accompagnent chaque famille. L'intensité des débats à la Chambre et la politique subtile des ministères Richelieu et Decazes obligent les trois principales forces à définir et préciser les priorités de leur conduite, tant au niveau des principes à défendre que des pratiques à mettre en place.

Cette fois, Bazin décrit « le parti ultraroyaliste, le parti ministériel et le parti libéral ou pour mieux dire, constitutionnel $»^{50}$. Les deux premiers sont présentés de façon péjorative : l'un aurait pour devise «Dieu, le roi et les dames, mais surtout vivent les prêtres et les Bourbons ", tandis que le second est clairement perçu au travers d'un opportunisme politique qui fait de ses avocats «les hommes inébranlables qui, à travers toutes les révolutions, se sont tenus, avec une constance digne des stoïciens les plus intrépides, du côté des pensions, des honneurs et des places ». En revanche, le parti constitutionnel est composé de personnes qui veulent « demeurer libres sous un gouvernement représentatif, [...] Il leur importe d'ailleurs assez peu que tel homme soit ministre plutôt que tel autre, pourvu que celui qui l'est, soit obligé de gouverner dans un sens national... ${ }^{51}$.

20 L'énoncé de ces trois forces ne suffit pas à la constitution d'un champ politique fonctionnant de façon légale et autonome. Pour Bazin, plus que cette division tripartite des forces politiques françaises, c'est la vitalité de l'opposition constitutionnelle qui légitime l'ensemble des institutions. Il retrouve, dans ces pages, le cheminement des républicains démocrates de l'anIII et des années suivantes. À cet effet, en 1816, il avance deux notions clés: l'opinion publique et l'opposition constitutionnelle... La première «est le contrepoids de l'autorité publique. Elle se forme du concours des opinions particulières et sort de la conscience du Peuple.... Ses jugements de première instance ne sont pas toujours dictés par la droite raison ; mais elle ne manque pas de les rectifier avec le temps, car elle seule a le pouvoir de réviser, de modifier et de casser ses propres arrêts... L'opinion publique est un flambeau qui éclaire la conduite et la marche des gouvernements $\|^{52}$. La seconde est "la résistance constitutionnelle du parti populaire au parti des ministres... Les partis se forment dans une révolution entreprise par l'esprit de liberté, et se régularisent après que cette révolution est finie. Le besoin de repos fait naître un pacte en vertu duquel le principe de gouvernement est modifié ou changé ; mais le parti qui s'était opposé à ce changement reprend la direction des affaires, tandis que le parti contraire garde la direction de l'opinion publique : ainsi se forment le ministère et l'opposition $"^{53}$.

21 Tel un organisme, la politique est mue par des forces différentes. Aussi, «faut-[il] laisser à la machine politique le contrepoids de l'opposition... Se soumettre au régime constitutionnel revient à accepter l'union de la liberté et de la monarchie; seule la résistance constitutionnelle est légitime face à l'emploi de la force $»^{54}$. Ainsi l'exposé des règles du champ politique exprime un jeu de forces : une " tension équilibrée » naît du conflit entre les partis politiques: autant de preuves tangibles des libertés, de l'égalité, de l'alternance, voulues, garanties et protégées par une Constitution qui devrait être approuvée par le Peuple. Bazin a soin de rappeler la mauvaise réputation du mot « parti $»^{55}$. «On a regardé comme alarmant d'avoir annoncé en France un parti d'opposition. Un tel reproche décèle peu d'habitudes constitutionnelles... Sous un gouvernement libre, il y aura toujours une opposition, toujours des partis dont chacun parlera, écrira dans le sens qui lui est propre, mais ne s'agitera point pour changer la 
forme de ce gouvernement: ils se renfermeront et se balanceront dans le cercle constitutionnel $\aleph^{56}$. Il importe à Bazin, reprenant les propos du ministre Decazes sur la liberté de la presse, de montrer que les forces politiques sont structurées en partis, devenus une réalité tangible de la vie parlementaire et de l'opinion nationale ou locale. Ainsi l'élargissement $\mathrm{du}$ corps électoral et la formation de cercles de réflexion représentent deux moyens préconisés et défendus pour insuffler une dynamique civique, capable d'offrir une légitimité réelle au régime né de l'effondrement de l'Empire.

Devant l'impossibilité de demander des scrutins organisés au moyen d'une participation populaire, l'enjeu politique, au début de l'année 1817, se porte sur l'organisation des collèges électoraux qui doivent « sortir du sein de l'industrie » et des villes, seul moyen de parvenir à "la monarchie représentative $»^{57}$. Conscient des restrictions imposées par la Charte dans l'article 38, définissant les conditions exigées pour devenir électeur, Bazin en suggère une possible révision et souligne en revanche, l'opportunité offerte par l'article 53, de pouvoir rédiger des pétitions en cas de nonaccord sur le mode de rétribution des curés de paroisse. Ces questions adressées à la Charte mettent en valeur l'actualité des problèmes, posés de façon lancinante depuis l'été 1789. En 1817, dans la nouvelle donne constitutionnelle, quelle place politique est accordée au Tiers État pris dans son sens le plus large?

Dans les provinces, la vie politique est encore réservée à une minorité. Loin de Paris, il devient difficile d'organiser d'authentiques partis politiques. Les opinions peuvent s'exprimer pourtant, hors des périodes électorales, dans des cercles littéraires, capables d'organiser une sociabilité politique et pacifique, où la culture et la critique positive du gouvernement doivent concourir à l'édification d'une conscience civique. C'est ainsi qu'il faut comprendre l'histoire "de la raquette ", histoire à peine codée, inventée par Bazin. Dans une ville, des jeunes gens ont formé une «Société libre de la Raquette » où l'on joue au volant. Des gens, à l'affût de tout, dénoncent sans tarder « la Conspiration de la Raquette ", qui ne serait, selon eux, qu'une épouvantable société libre, animée par une trentaine de fous armés de raquettes. Le commissaire les arrête... pour rien ; si ce n'est pour déclencher le rire inextinguible des soi-disant comploteurs. "Redevenus calmes, ils [les jeunes] se réunirent pour traiter, avec tout le sang-froid imaginable, la question très neuve de la liberté individuelle... Rien ne transpira, du moins hors de l'enceinte, des délibérations $»^{58}$. Il fut décidé alors que la réunion se poursuivrait par une réflexion sur l'héritage de la philosophie du siècle précédent. La morale de cette affaire apparaît clairement : le climat de suspicion au niveau local ne peut que pousser les forces vives du pays à se réunir afin de mettre en perspective des projets de transformations politiques ${ }^{59}$.

L'éducation, mère de la saine politique

Bazin a la conviction, comme bien d'autres, qu'aucune mesure politique, aucune loi ne saurait avoir une réalité et une efficacité quelconque chez un peuple ignorant, parmi une population dont les mœurs, les coutumes n'auraient pas intégré les conditions préalables au changement imposé par les institutions ou la législation. La discussion dans les cercles locaux de l'importance de Voltaire ou de Rousseau, doit symboliser ce rapport entre la construction politique et le partage d'un savoir, nouveau ciment d'un contrat social hérité de la Révolution. Ici, ce n'est pas tant la reconstruction a posteriori d'une philosophie des Lumières ayant engendré la Révolution qui est pertinente, mais bien plutôt le constat, par la réaction de rejet qu'elle provoque dans le camp des 
conservateurs, de la charge politique contenue dans l'œuvre de Voltaire et de Rousseau, frères ennemis et "pères fondateurs" de 1789. Dans une dynamique de mouvements opposés, le message descendant des philosophes rencontre un mouvement ascendant, caractérisé par cette volonté d'apprendre du peuple, que symbolise pour Bazin l'alphabétisation des campagnes, enjeu politique fondamental dès le début de la Restauration.

Ainsi, Pierre, le paysan, figure de proue de nombreuses historiettes imaginées par Bazin, résume de façon vivante ce débat érudit et polémique. Ancien maire de sa commune pendant la Révolution, rentré dans le rang, travailleur, bon père de famille, mû par un besoin de comprendre, il possède un bon sens redoutable. Il se voit flanqué d'autres figures types de la société, le comte propriétaire, homme d'esprit, résolument ouvert et philanthrope, jamais avare d'explications, et monsieur Roquentin, l'ancien bailli d'avant 1789 , bourgeois obstinément réactionnaire. Ce dernier ne peut que s'élever contre des campagnards qui veulent savoir lire et écrire, devenant ainsi des « demi-paysans qui défendraient les paysans comme les demi-bourgeois se sont élevés pour défendre le peuple», en $1789^{60}$. Deux logiques s'affrontent. Monsieur Roquentin tente d'établir le lien entre l'alphabétisation des paysans et les guerres de la Révolution et de l'Empire: "Apprenez à lire à vos marmots et ils voudront tous sortir de leur village pour devenir maréchaux de France $\aleph^{61}$. N'est-ce pas là une inquiétude sociale qui a dû être réelle? La promotion des paysans sachant lire, en soldats, constituait à terme, dans l'État, une république de militaires, peu ou prou savante, résolument belliqueuse, et qui risquait d'entraîner le pays dans des dangers intérieurs et extérieurs à éviter en cette période d'humiliante occupation militaire étrangère. Monsieur Roquentin en tire les conclusions et refuse de passer un nouveau bail avec son fermier qui envoie ses enfants à l'école.

Pierre explique bien autrement la nécessité de l'alphabétisation. D'abord, parce que les paysans doivent apprendre à se défendre eux-mêmes, ensuite, parce que «le sort des laboureurs s'est bien amélioré depuis vingt-cinq ans, beaucoup d'entre eux sont devenus des propriétaires ». Ils n'ont donc aucun intérêt à aller faire la guerre à l'étranger. Les paysans vont, enfin, «se mêler de leurs affaires et bientôt il y aura une école à côté de chaque presbytère "... Et Pierre d'ajouter : « C'est un fier aiguillon que ce petit mot de liberté ! $»^{62}$. Le comte, allié objectif du paysan conclut : « Les prêtres et les poètes sont les instituteurs des peuples enfants; les historiens et les philosophes sont les instituteurs des peuples mûrs ${ }^{63}$, liant encore une fois la figure du philosophe à celle du peuple à éduquer.

Dans cette perspective, les deux figures qui s'imposent sont Rousseau et Voltaire et permettent à Bazin de passer du problème de l'éducation à celui de la formation de l'opinion. Un avocat, chargé de défendre les deux philosophes devant la "Société de la Raquette", rappelle, en ce printemps 1817, l'erreur qui rend les philosophes responsables des événements de la fin du XVIII ${ }^{e}$ siècle, là où ils n'ont fait que traduire une évolution plus profonde de la société. "Tant qu'une forme de gouvernement satisfait aux désirs du plus grand nombre, elle se maintient contre le reste ; aussitôt que ces besoins s'accroissent par une plus grande aisance et par une instruction plus étendue, ils sont contrariés par les classes privilégiées ; l'opposition entraîne des débats polémiques avant-coureurs des querelles politiques ; les besoins cherchent à s'étayer de l'autorité du raisonnement et de l'exemple; on appelle l'histoire, l'imagination, la philosophie, au secours de l'innovation. C'est alors que des écrivains féconds, brillants, 
véhéments, hardis, créent une opinion publique et s'en font les tribuns ; [...] Les écrits ne sont donc que des instruments dans les révolutions, et lorsqu'on s'occupe du soin de les réprimer, il est déjà trop tard: on croit avoir saisi la cause; on ne tient que l'effet $\aleph^{64}$. L'auteur donne une réponse toute en subtilité sur la filiation entre Lumières et Révolution. Loin de l'avoir provoquée ou anticipée, la philosophie n'a fait que constater, après-coup, la révolution physique qui s'était produite dans le corps social, dont les transformations politiques n'ont été que des manifestations ultérieures. La politique de Bazin se présente comme une réflexion globale sur les ressorts qui lient esprit public, essence du régime et fonction de la culture politique, comme traduction et régulation des différentes composantes du corps social.

L'Histoire à la rescousse des républicains

À l'intérieur du système de réformes induites par l'adaptation cohérente de la Charte constitutionnelle à la nation, Bazin accorde enfin, une importance particulière aux conditions historiques qui ont rendu cette situation possible dans le pays, car elles seules expliquent cet apparent paradoxe d'un texte enfin constitutionnel dans une France monarchique. Bazin assume là ce legs de la philosophie des Lumières, liant la recherche de la causalité à la critique politique, dans la perspective optimiste de restituer le temps à tous ses acteurs, devenus capables de façonner le réel à leur volonté65. Cet héritage est invariablement lié à Voltaire, si souvent cité dans Le Lynx. En racontant l'Histoire à travers le prisme de la Révolution, le sens des événements en ressort transformé. Le temps abordé de façon téléologique va induire un discours que le XIXesiècle ne cessera d'alimenter ou de critiquer : la décennie révolutionnaire apparaît comme la fin d'une histoire ancienne et le début d'une histoire moderne.

Le récit du temps passé équivaut à une longue émancipation du Tiers État se libérant de tutelles oppressives et successives où la dépendance économique de la multitude est clairement utilisée dans une optique politique. Aussi n'est-on pas peu surpris de lire que « les anciens gaulois, jouissaient presque de la liberté » car ils entretenaient entre eux des rapports de "patrons à clients ». Le régime féodal, venu du nord, a rompu ces relations et a imposé des maîtres aux esclaves. Cependant, comme le travail enrichissait ces derniers, ils se réunirent en communes et en corporations. Les Parlements et les Universités furent alors constitués en corps intermédiaires, pendant que les États discutaient les impôts. "En un mot, tous les changements qui s'opéraient dans nos institutions étaient au profit du Tiers État, dont les rois avaient besoin pour réprimer l'insolence de leurs vassaux ${ }^{66}$. Cependant, la ruine des fiefs allait faire du roi «le seul patron du peuple ». Dès lors la monarchie prit tout son essor vers une souveraineté absolue et lorsque « les Parlements osèrent lever la voix contre ses édits, la nation fut avertie qu'une seule volonté faisait la loi en France ». C'est cette situation que la Révolution a bouleversée. "De droit et de fait, toute espèce de patronage était anéantie ; mais la force réelle, prise dans le sens physique et moral du mot, étant alors du côté du Tiers État, il fut bientôt résolu qu'au despotisme succéderait le patronage des lois. Et depuis 27ans, la France, l'Europe, le monde entier se meuvent par l'effet de cette résolution $»^{67}$. L'intérêt, ici, est de penser les liens entre les modalités de la domination politique et les termes de la domination économique, dans la formation des différents régimes, et de mettre en évidence la relation de patronage à l'origine du lien social... puisque, en fin de compte, la Révolution n'a pas supprimé cette relation, mais a fait de la loi « la patronne " des relations civiles... ce qui en soi devrait interdire de penser la Révolution comme un simple transfert de souveraineté absolue du roi au 
peuple : cette notion de patronage introduit alors une dimension complexe dans la construction du rapport politique.

Pour autant, la question demeure du subit dérèglement de la monarchie au XVIIIesiècle et de la recherche des causes de la Révolution. Il s'agit de trouver dans les dysfonctionnements de l'État royal, les raisons autonomes de son discrédit, avant de prétendre que la crise d'Ancien Régime a été une invention du parti des philosophes: «Était-ce la philosophie qui animait Richelieu contre les protestants et les nobles ? Estce la philosophie qui animait madame de Maintenon contre les protestants et les jansénistes ? Est-ce la philosophie qui créait à la mort de Louis XIV, une dette publique d'un milliard quatre cents millions? Fut-elle pour quelque chose dans la fameuse banqueroute de Law ? La vit-on assise sur le trône, ou même sur les marches du trône, sous le règne de Louis XV? Madame de Pompadour était-elle philosophe? Enfin Calonne, le cardinal de Rohan et le déficit étaient-ils philosophes? Les voilà pourtant les causes de la Révolution ${ }^{68}$. Ce n'est pas tant le degré de véracité et d'objectivité du témoignage qui intéresse que ce qu'il révèle de la sensibilité d'un républicain démocrate écrivant sous la Restauration et des catégories historiques convoquées et constituant le fondement de la pensée historique des républicains pour tout le siècle ${ }^{69}$. L'absolutisme ou le pouvoir arbitraire sont finalement moins sévèrement dénoncés que la crise financière sans cesse rappelée dans les dettes de l'État.

31 À ce titre, Bazin émet une hypothèse intéressante sur le rôle de la noblesse dans la chute de l'Ancien Régime. Après avoir consacré de longs paragraphes à l'ensemble des révoltes que la noblesse avait organisées pour résister à l'arbitraire de la monarchie, il finit par conclure: «Observons seulement que dans le siècle dernier, les prétentions féodales de la noblesse avaient disparu, et que la liberté s'est inoculée chez nous par le contact des jeunes gentilshommes, frères d'armes de Lafayette, élèves de Washington, héros de l'indépendance aux États-Unis d'Amérique ${ }^{70}$ ». Lucide dans sa volonté de présenter l'histoire de la noblesse, et non son roman, comme il le reproche à Chateaubriand, il insistait sur le rapport entre les révolutions antérieures anglaises et américaines et la Révolution française.

La nécessité de penser ensemble les révolutions anglaise, américaine et française

À ce point de la démonstration, la réflexion sur le Directoire permet à Bazin de lier les trois bouleversements politiques et d'émettre, par-delà les poncifs éculés sur la République corrompue, instable, et le prétendu désintérêt du peuple, une hypothèse originale sur l'échec du régime né de la Constitution de l'an III : «Après avoir aboli le Directoire, Bonaparte dit à la France qu'elle était mal gouvernée : rien de plus vrai ; mais la nation fut-elle avilie sous ce mauvais gouvernement? Malgré les défaites de Schérer, les troubles de l'Ouest et du Midi, la division des chefs de l'État et les dissensions des représentants, l'esprit de liberté ne s'affaiblit point; il respira dans les actions, dans les écrits, à la tribune, dans les camps, au sein du peuple; en un mot, la nation, abandonnée de son gouvernement, continua de se soutenir contre tous ses ennemis; et l'homme qui se saisit du pouvoir au 18Brumaire était le premier général d'Europe. Si la France eut son Cromwell, elle croyait posséder son Washington ${ }^{71}$. À force de comparer Robespierre ou Bonaparte à Cromwell, certains avaient perdu de vue l'autre Révolution du monde atlantique... ou plutôt, ses prémices, son déclenchement, étaient-ils étudiés, mais sa fin était rarement mise en rapport avec le 18 Brumaire. Au moins, le parallèle établi par Bazin a-t-il eu le mérite d'expliquer le possible malentendu entre la nation et son général victorieux et toujours républicain au soir du 
19 Brumaire ${ }^{72}$. En refusant, en toute conscience, d'assumer le «moment américain de l'Histoire de France ", Bonaparte niait la leçon de la Révolution de 1688 et ses conséquences jusqu'en 1776, et préparait, bien avant la Restauration, les conditions nécessaires « au moment anglais de l'Histoire de France».

Plus tard, à la fin de l'ouvrage, Bazin développe ce parallèle obligé pour les contemporains : «En Angleterre, la restauration des Stuarts, semblable à celle des Bourbons, ne produisit que deux règnes: la postérité de Jacques II serait peut-être encore sur le trône britannique, si ce prince eût ressemblé à Louis XVIII, et s'il eût reconnu les droits de ses sujets $»^{73}$. Pourtant, l'Histoire s'impose telle la source première de légitimité du politique. Ce n'est donc plus une simple métaphore transformée du présent qu'offre l'Histoire mais sa faisabilité réelle. Bazin l'exprime en liant la monarchie constitutionnelle à la Charte, à son tour dépendante de son expression pratique: le gouvernement représentatif. La légitimité, la défense de la liberté et la souveraineté nationale - c'est tout un pour Bazin - reposent sur la manifestation ancienne de la volonté du peuple. «Il faut toujours remonter à cet acte pour trouver le vrai titre de la légitimité. Autrement, quelle serait la garantie des rois contre les prétentions du Saint Siège... ? Pépin-le-Bref ne mit la couronne sur sa tête qu'après avoir consulté le vœu des Français. Hugues Capet n'obtint d'abord que le suffrage de ses propres vassaux réunis en armes dans l'église de Noyons; mais ensuite, la majorité des grands feudataires légitima ce couronnement par son adhésion ». La situation de 1817 est donc le résultat entremêlé de deux histoires, celle de la monarchie et celle de la souveraineté du peuple : «Le roi peut gouverner sans le peuple, mais non sans les représentants [...] Le prince jure de se conformer à une Charte constitutionnelle, irrécusable de la souveraineté du peuple, prise dans le sens réel et philosophique des choses $\gg^{74}$... Raisonnablement, Bazin ne peut pas, au risque évident de la censure et de l'emprisonnement certain, pousser jusqu'au bout les conséquences de sa démonstration politique. Il faut donc attendre les derniers fascicules du recueil, Coup d'œeil sur le système européen et dernier coup d'œeil, pour entrevoir la récapitulation audacieuse de son projet. Ce que le rappel du passé lui interdisait, à savoir la formulation d'une républicanisation de la monarchie, la géopolitique et la projection dans le futur politique de l'Europe vont le lui permettre en toute dernière analyse, et de façon éclatante.

L'Europe, futur de la République?

De fait, Bazin demeure sous le charme de la prose de Saint-Simon (SSdans le texte) qu'il lit dans les colonnes du Censeur. Il cite longuement De la Réorganisation de la Société Européenne, ou de la Nécessité et des Moyens de Rassembler les Peuples d'Europe en un Seul Corps Politique, en Conservant à chacun son Indépendance Nationale, de $1814^{75}$.

35 En somme, "l'Europe aurait la meilleure organisation possible si toutes les nations qu'elle renferme, étant gouvernée chacune par un Parlement, reconnaissaient la suprématie d'un Parlement général placé au-dessus de tous les gouvernements nationaux, et investi du pouvoir de juger leurs différends... Dès l'instant qu'une nation aura adopté la forme du gouvernement représentatif, elle [pourra] s'unir à la société et députer au Parlement commun des membres pris parmi elle, et l'organisation de l'Europe s'achèvera insensiblement sans guerres, sans catastrophes, sans révolutions politiques... ${ }^{76}$. Encore une fois, Bazin appuie sa démonstration sur la série des faits observés lors des révolutions anglaise et française. Ayant des termes en commun, elles auraient le même avenir. «M.deSS propose de rendre complète la ressemblance entre 
notre Constitution et celle d'Angleterre, en séparant la royauté de l'administration ${ }^{77}$. Enfin, pour que l'ébauche de la construction politique de l'Europe fût achevée, il faudrait hâter la réorganisation de l'Allemagne par la transformation pacifique de ses institutions. Novateur s'il en est, le projet pose clairement les bases d'une république anglo-française dans un premier temps: lucide et somme toute sceptique, Bazin termine son résumé par cette interrogation: «La confédération européenne est-elle possible?»

Inversant un rapport supposé évident, celui qui est présenté comme un utopiste jacobin, un faiseur de systèmes démocratiques dangereux, Bazin, soulève quelques problèmes de politique pratique, intéressants à identifier afin de comprendre la validité d'une construction possible d'une république de Parlements européens. Trois types d'obstacles sont distingués : historique, politique et diplomatique.

Historiquement d'abord, «le moi » d'une nation s'est construit, comme l'écrit Bazin, selon un partage de sentiments, de consanguinité, de sol, de climat, de communauté de langue, qui créent le lien politique qu'est une nation : «vouloir étendre au-delà le lien politique, c'est vouloir le briser...» et prendre le risque d'ôter aux nations leurs différences, ce qui les mettrait sous la coupe d'un seul maître. Ainsi la confédération des nations aurait écarté le danger de la guerre mais se «dessécherait sous le despotisme ». "Imaginer de transformer l'Europe en un seul corps dont un grand parlement serait la tête, c'est conseiller aux nations de se démettre enfin du droit géant, dit-on, d'exercer leur intelligence et leur volonté ? $\aleph^{78}$. Politiquement ensuite, le risque encouru par un Parlement européen est de voir se constituer, au nom de la liberté, une institution exécutive dont les prérogatives empêchent tout contre-pouvoir de se manifester. Comment, dès lors, former une opinion publique européenne capable d'influer vraiment sur les décisions des députés dont le pouvoir est infiniment plus facile à organiser? Car enfin, et c'est le troisième point des observations de Bazin, imaginer une construction européenne sur la parité des relations entre l'Angleterre et la France, revient à refuser de prendre en compte la politique extérieure de l'Angleterre, construite sur la claire perception que sa puissance est due à la faiblesse de la France : «Elle croit que nous avons manqué notre Révolution et qu'en élevant par la liberté, les nations continentales au-dessus de la nôtre, elle nous condamne à demeurer le dernier peuple de l'Europe $»^{79}$.

Il revient donc au révolutionnaire de rappeler quelques éléments de Real Politik au penseur libéral... dont le projet économique est également critiqué : «La guerre, les orages politiques, les vicissitudes du despotisme et de la liberté, valent mieux que le calme (fort imaginaire du reste) que l'on ferait naître de toutes les facultés humaines dirigées vers l'industrie. On appelle les soldats, des machines à fusil; et que sont la plupart des ouvriers ? si ce n'est aussi des machines $»^{80}$. Bazin émet une considération plus modérée mais résolument perspicace : il finit par comparer la situation de deux Europes. La première tournée vers ce qu'il appelle la réaction, présente tous les stigmates dangereux du passé. «L'objet de la seconde est d'extirper de l'Europe trois grands fléaux : la guerre, l'arbitraire, le monopole; de faire que, dans tous les pays, on puisse exercer librement toute espèce d'industrie utile, et être assuré d'en recueillir les produits; enfin, d'introduire les formes de gouvernement les plus propres à garantir ces avantages et à les garantir à moins de frais possibles $»^{81}$. Une fédération des nations européennes n'est plus impossible. Cohérente, elle devient la traduction des récentes transformations de l'industrie, et de l'expérience révolutionnaire, de part et d'autre de 
la Manche, faisant du gouvernement représentatif la forme adéquate de la modernité politique... Ou, comment penser sans l'écrire une république européenne...

Ainsi, la pensée de Bazin apparaît plus riche et plus complexe. Le porte-plume sarthois, loin des sources d'informations parisiennes, sans cesse contrôlé par la police mancéenne, fort d'une réputation exécrable de Jacobin fauteur de troubles, mais conscient du réseau républicain et de son influence dans ce département «bleu», délivre une pensée, plus en nuances qu'il n'y paraît, capable d'intégrer les nouvelles contraintes du jeu politique. La Charte est admise ainsi que la monarchie : il s'agit donc d'en appliquer le principe, avec la claire perception des bouleversements intervenus en France depuis vingt-cinq ans.

Tout au long de ses quatre cents pages, et pour peu que soit dépassé le ton polémique, Bazin dévoile une pensée d'une lucidité politique réelle. Totale dans sa conception, sa réflexion pose de façon pertinente des liens constitutifs entre l'Histoire, l'organisation des institutions, le fonctionnement de l'économie, et l'éthique qui en découle. La politique est une science du social qui laisse entrevoir le projet de Bazin - indicible, et pour cause -, mais qui apparaît entre les lignes : comment faire que le régime né de la Charte constitutionnelle aille au-delà de la monarchie représentative pour s'adapter à la forme toute nouvelle d'une République royale?

41 Bazin perçoit une issue politique qui aurait rendu la Restauration possible, qui aurait placé, du moins, les libéraux et les anciens révolutionnaires de son côté. L'adaptation républicaine de la monarchie semblait, à partir de là, une option raisonnable. C'était sans compter sur la faculté de résistance d'une part importante de la société, qui pensait sincèrement que la source des maux subis par la France depuis 1789, venait de " ces abus innombrables " perpétrés par tous ceux qui se réclamaient du parti de la liberté. Dès lors, la pensée de Bazin était vouée à l'échec: le plomb du pistolet d'un jeune hobereau échauffé par les sarcasmes antinobiliaires de celui qui aimait à se définir comme un « écrivain constitutionnel » allait frapper au cours d'un duel, une vie tout entière vouée à l'idée républicaine, fût-elle transposée dans le cadre d'une république monarchique au sein d'une fédération des nations européennes ${ }^{82}$.

42 La France avait approché "son moment américain » et l'avait gâché ${ }^{83}$; mais la leçon républicaine n'est pas restée lettre morte. Goyet, l'ami fidèle depuis 1793, allait reprendre le flambeau et poursuivre la diffusion des idées radicales dans Le Propagateur qui, en défendant les acquéreurs des biens nationaux, attirait la sympathie des électeurs inquiets des prétentions du clergé et de la noblesse. Aussi, les élections de 1818 et 1819, allaient-elles faire de la Sarthe un "îlot républicain ", avec ses quatre députés, libéraux et ouverts aux idées républicaines. Benjamin Constant en faisait partie, mais surtout l'accompagnait celui qui fut en son temps, le jeune défenseur de la République du nouveau monde: La Fayette ${ }^{84} \ldots$ alliance posthume paradoxale, ou lointaine origine de l'opportunisme républicain? 


\section{NOTES}

1.Roger CHARTIER, Les Origines culturelles de la Révolution française, Paris, Seuil, 1992. KeithBAKER, The French Revolution and the Creation of Modern Political Culture, vol.1; The Political Culture of the Old Regime, Oxford 1987, et Au Tribunal de l'Opinion, Paris, Payot, 1991.

2.Jean-François SIRINELLI, « L'Histoire politique et culturelle », Sciences Humaines, septembre-octobre 1997, n 18, pp.36-39.

3. Voir Serge ABERDAM, Serge BIANCHI, Robert DEMEUDE, Émile DUCOUDRAY, Bernard GAINOT, Maurice GENTY, Claudine WOLIKOW, Voter, Élire, pendant la Révolution française, 1789-1799. Guide pour la Recherche, Paris, CTHS, 1999.

4. Voir Timoty TACKETT, Par la volonté du peuple. Comment les députés de 1789 sont devenus révolutionnaires, Paris, Albin Michel, 1997, et Keith BAKER, The Political Culture of the Old Regime, in the French Revolution and the Creation of modern Political Culture, Oxford 1987 et Pergamon Press, 1987, vol.I.

5.Étudiant les idées politiques des groupes modérés ou libéraux, Pierre Rosanvallon consacre peu d'importance à la théorisation de la démocratie représentative durant le Directoire, rendant plus difficile la compréhension du lien entre la génération des républicains de la Restauration et son regard sur la Constitution de l'AnIII. Cf.Pierre ROSANVALLON, La Démocratie inachevée. Histoire de la Souveraineté du Peuple en France, Paris, NRF, Gallimard, 1999, pp.86-91 et 97-99. Toute l'œuvre d'Isser WOLLOCH, et plus particulièrement The New Régime, Transformations of the French Civic Order, 1789-1820, New-York, Norton and Company, 1994, suffit à montrer l'importance de l'expérience politique directoriale dans l'histoire du XIXe siècle. Sur l'aménagement possible de la Constitution de l'An III en démocratie représentative, qu'il me soit permis de renvoyer à mon Antonelle, Aristocrate révolutionnaire, Paris, Felin, 1997.

6. Les Pamphlets de la fin de l'Empire, des Cent Jours et de la Restauration, catalogue raisonné, mis en ordre par Léopold A.-G. GERMOND DE LA VIGNE, Paris, 1879.

7.Pierre ROSANVALLON, La Monarchie Impossible. Les Chartes de 1814 et 1830, Paris, Fayard, 1994. Introduction: « Les Deux Histoires de France ", p. 8.

8.L'expression est usitée dans le journal républicain, Le Nain jaune, réfugié à Bruxelles, IIIevolume, année 1816, p. 480.

9.Le parcours de Bazin est à placer dans le contexte d'un département fortement marqué par l'expérience révolutionnaire qui en fait, et pour longtemps, une terre hautement républicaine. Cf.ChristinePEYRARD, Les Jacobins de l'Ouest, Paris, Publications de la Sorbonne, 1996.

10.Eugenio DI RIENZO, Marc-Antoine Jullien de Paris, una Biografia politica, Napoli, Guida editori, 1999 et Isser WOLOCH, Napoléon and his collaborators. The making of a dictatorship, New York, Norton et Company, 2001.

11. Marcel REINHARD, Le Département de la Sarthe sous le Régime directorial, Rennes, Les Presses bretonnes, 1936.

12.R. BAZIN, Le Démocrate ou le Défenseur des Principes, R.F. Bescher et J.R. Bazin, 12 fructidor, anVII, B.N.F. Lc2 2741. Cf. Christine PEYRARD, « Combats parisiens pour la démocratie de journalistes de l'Ouest », Paris et la Révolution, Michel Vovelle (dir.), Paris, Publications de la Sorbonne, 1989, pp.321-322.

13. Cf. Jacques Rigomer Bazin, (1771-1818). Itinéraire d'un révolutionnaire démocrate. Mémoire de maîtrise de Charlotte LECONTE, sous la direction d'Annie Duprat, Université du Maine, 2000.

14.Dès fructidor anVII, Briot évoque, pour la première fois, le « Parti des républicains indépendants ». Cf. Bernard GAINOT, 1799, un nouveau Jacobinisme?, Paris, CTHS, 2001. En brumaire anVIII, Antonelle, alors rédacteur en chef du Journal des Hommes Libres, imagine à son tour l'alternance des partis conservateurs et démocrates par le jeu régulé et accepté des élections annuelles. Cette définition des partis permet de rompre une logique qui considère l'organisation politique structurée comme un élément de parasitage entre le représenté et son mandant, et démontre, de la part de la gauche directoriale, une capacité à s'organiser publiquement dans une perspective des élections qui dépasse la logique du club ou de la section. De façon significative, les démocrates de l'anVII militent en faveur d'un suffrage sans cesse élargi, de la liberté de la presse, des 
opinions et des pratiques religieuses - pourvu qu'elles soient privées -, de la nécessité de l'instruction élémentaire assurée et du droit de pétition.

15. «Tant que les hommes qui gouvernent auront la prétention de s'opposer aux progrès de l'esprit humain, en ce sens qu'ils voudront entraver sa marche vers son but unique, le bonheur de l'humanité, il se formera nécessairement une opposition qui sera en raison des lumières du peuple qu'ils opprimeront » Bazin, Le Démocrate, $\mathrm{n}^{\circ}$ 27, 12 fructidor an VII.

16.Archives de la Préfecture de Police de Paris (A.P.P.), Aa316, Rapport général de l'Affaire Malet, 1808.

17.Rigomer BAZIN, Le Lynx, B.N.F. Lb48 973. « Seïde. Ma Vie est un Combat », p.9.

18. Rigomer BAZIN, Les Lettres Philosophiques, Paris, chez l'éditeur, rue d'Aboukir nº 31, 1807, p.10, B.N.F. Z 15354.

19.Ibid, p.343.

20.Peut-être est-ce l'indice qui confirme l'attribution par BARBIER, de L'Histoire des sociétés secrètes de l'Armée, et des Conspirations militaires qui ont eu pour objet la destruction du gouvernement de Bonaparte, parue en 1815, à NODIER, LEMARE et à BAZIN., dans Dictionnaire des ouvrages anonymes et pseudonymes, Paris, 1822-1827, 4 vol., II, p.768.

21.Cf. l'article de Jean DAUTRY, « La Police impériale et les Révolutionnaires », Glanes, AHRF, 1968, n¹94, article réalisé à partir des Archives de la Préfecture de Police de Paris, Aa 316, op. cit.

22.R.BAZIN, Les Lettres Philosophiques, Paris, rue Saint-Sauveur, $n^{\circ} 6$, de l'imprimerie de Renaudière, 1814, Première Lettre, B.N.F. Lc2 2779

23.R.BAZIN, Le Lynx, Coup d'Eil et Réflexions Libres sur les Écrits et les Affaires du Temps, recueil publié par M. Rigomer Bazin, Paris, Blanchard, libraire Galerie Montesquieu, janvier 1815, B.N.F.Lb48 973 (bis).

24. Constatant la diversité des interprétations du texte de 1814, Stéphane Rials conseille « d'admettre la Charte telle qu'elle fut pensée, même si ce pensé ne fut pas toujours cohérent, même si le brouillage des lectures contradictoires est parfois malaisé à surmonter... » (Stéphane RIALS, Révolution et Contre-Révolution au XIXe siècle, Albatros 1987, p.94).

25. Madame DE STAËL, Considérations sur la Révolution française, CEuvres complètes, Paris, 1820, XIII, pp.154-155.

26. Cf. Eugenio DI RIENZO, L'Aquila e il Berretto grigio, per una Storia del Movimento democratico in Francia da Brumaio ai Cento Giorni, Napoli, Edizioni Scientifiche Italiane, 2001

27. Emmanuel DE WARESQUIEL et Benoît YVERT, Histoire de la Restauration, 1814-1830. Naissance de la France Moderne, Paris, Perrin, 1996 et G. BERTIER DE SAUVIGNY, La Restauration, Paris 1990

28.A.N., F. 18/21.

29. Pour la seule année 1816, il est possible de citer Mon Procès, B.N.F. Lb48 785; Le Trône et l'Autel, ou Réponse à Monsieur de Chateaubriand par un ci-devant Révolutionnaire, B.N.F. Lb48 2939; Doutes éclaircis par un constitutionnel, B.N.F. Lb48 656; Charte expliquée aux habitants des campagnes, Lb48 647.A; Lettre à un révolutionnaire d'aujourd'hui par un révolutionnaire d'autrefois, Lb48 725.

30. Lettre du préfet de la Sarthe au ministre et datée du 21 septembre 1816, A.N. F. 18/21.

31.Ibid, lettre du 26 septembre 1816.

32.Cf. Christine PEYRARD, « Le journalisme et la diffusion de l'idée républicaine dans la Sarthe, de la Première à la Seconde République » dans Révolution et République, l'Exception française, sous la direction de Michel Vovelle, Paris, Kimé, 1992, pp. 511-521.

33. Lettre du 21 novembre 1816.

34.R. BAZIN, Mon Procès, Première Partie, pp.194-228.

35.R. BAZIN, Mon Procès, Deuxième Partie, pp.230-266.

36.R. BAZIN, Mon Procès, p.240. « La loi d'élection du 5 février 1817 est sans doute l'un des événements politiques les plus importants de l'histoire de la Restauration. Elle instaure un nouvel ordre politique et social et peut être considérée avec Rémusat, comme "l'acte d'investiture de la classe moyenne comme classe gouvernante" » (tous les contribuables âgés de plus de trente ans et payant plus de trois cents francs de contribution votent, les électeurs se réunissent au chef-lieu de canton en un collège unique. L'élection est directe: c'est l'avènement des électeurs capacitaires). E. DE WARESQUIEL et B. YVERT, Histoire de la Restauration, op. cit., pp.208-209. 
37.R.BAZIN, Pierre chez Roquentin, p.361. Pour le texte de la Charte, Cf. Jacques GODECHOT, Les Constitutions de France depuis 1789, Paris, Garnier Flammarion, 1970, pp.217-224.

38. R. BAZIN, Lettre à un révolutionnaire d'aujourd'hui par un révolutionnaire d'autrefois, B.N.F. Lb48 725, p.4.

39.R. BAZIN, Le Trône et l'Autel ou Réponse à Monsieur de Chateaubriand par un ci-devant révolutionnaire, pp.58-59.

40.R. BAZIN, Doutes éclaircis par un constitutionnel, seconde édition revue et corrigée, au Mans, chez l'auteur, rue Sainte Ursule, $\mathrm{n}^{\circ}$; à Angers, chez Hainault, libraire, place du pilori, 1817, p.47.

41.R. BAZIN, Catéchisme à l'usage des constitutionnels, suivi de Tout est Bien, au Mans, chez l'auteur, 1816.

42.Ibid, p.3.

43.R. BAZIN, La Charte expliquée aux habitants des campagnes, p.88.

44.Ibid, pp.6 et 7 .

45. À ce texte, en succède un autre, totalement anti-phrastique, dont le titre, Catéchisme impolitique à l'usage des petites maisons, ne laisse planer aucun doute. Procédé provocateur, il présente sous le masque de l'ironie, un contre-tableau d'une France n'ayant pas accepté l'esprit constitutionnel de la Charte, et le termine ainsi: « Qu'est-ce que l'opinion publique ? Une baliverne anglaise ! Qu'est-ce que l'anarchie ? C'est l'égalité des droits ! Qu'est ce que la tyrannie ? C'est une Constitution! ", Le Lynx, p.80.

46.R. BAZIN, Nouveaux Doutes éclaircis par un constitutionnel, Point de monarchie sans la Charte, p.119. Par là, Bazin réintroduit tout le débat politique du XVIII e siècle sur la légitimité de la représentation du Parlement venant tempérer le pouvoir exécutif. Cf. Catherine MAIRE, De la Cause de Dieu à la Cause de la Nation, le Jansénisme au XVIII ${ }^{e}$ siècle, Paris, NRF, 1998.

47.R. BAZIN, Le Trône et l'Autel, op. cit., p.56.

48. R. BAZIN, Dernier Coup d'œil, p.401.

49.R. BAZIN, Séïde, op. cit., p.16.

50.R. BAZIN, Des Partis en France, p.382.

51.Ibid, p.384.

52.Ibid, p.8.

53.Ibid, p.10.

54.R. BAZIN, Séïde, op. cit., pp.20-22.

55.R. BAZIN, Nouveaux doutes éclaircis par un constitutionnel, Point de Monarchie sans la Charte, op. cit., p.114.

56.R. BAZIN, Ma Défense devant la Cour royale d'Angers, p. 258.

57.R.BAZIN, Le Trône et l'Autel, ou Réponse à Monsieur de Chateaubriand par un ci-devant révolutionnaire, p.65.

58. R. BAZIN, Voltaire et Rousseau, ou le Procès des Morts, Première Partie, p.320.

59. Ce type de réunion permet de penser la conservation d'une culture républicaine souterraine qui apparaît encore mieux à la génération suivante. Cf. Jeanne GILMORE, La République clandestine, 1818-1848, Paris, Aubier, 1997.

60.R. BAZIN, Pierre chez Lui, p.290.

61.Ibid, p.298.

62.Ibid, p.299.

63.R. BAZIN, Pierre chez son Curé, p.309.

64.R. BAZIN, Voltaire et Rousseau ou le Procès des Morts, Première Partie, p.319.

65. Voir Jean-Marie GOULEMOT, Le Règne de l'Histoire, Discours historiques et révolutions, XVIIe-XVIII' siècle, Paris, Albin Michel, 1975, rééd. 1996.

66. R. BAZIN, L'Assureur constitutionnel, op. cit., p.274.

67.Ibid, p.276.

68. R. BAZIN, Le Procès des Morts, Deuxième Partie, op. cit., p.342.

69. Voir Sophie-Anne LETERRIER, L'institution des sciences morales : l'Académie des sciences morales et politiques, 1795-1850, Paris, L'Harmattan, 1995.

70.R. BAZIN, Le Trône et l'Autel, op. cit., p.55. 
71.Id., p. 61.

72. Déjà, dès le premier texte de l'ouvrage Séide, Bazin avait mis en parallèle les deux généraux Monk et Bonaparte, travaillant tous deux contre la République, mais le second pour l'établissement d'une dynastie nouvelle... (Séide, op. cit., p.5).

73.R. BAZIN, L'Assureur constitutionnel, op. cit., pp.277-278.

74.R. BAZIN, Lettre à un révolutionnaire, op. cit., p.14.

75.R. BAZIN, Coup d'œil sur le Système Européen, p.385. Il est à souligner que Saint-Simon (SS) figurait sur la liste des abonnés aux Lettres philosophiques, saisie par la police, en 1808.

76.R. BAZIN, Coup d'œil..., op. cit., p.388.

77. Ibid.

78. Ibid, p.394. Cf. Georges BENREKASSA, Montesquieu, la Liberté et l'Histoire, Paris, Le Livre de Poche, 1987.

79.Ibid, p.396.

80.R. BAZIN, Un Dernier Coup d'œil, p.410.

81.Ibid, p.411. Sur les fondements possibles de cette réflexion, voir Marc BELISSA, Fraternité universelle et intérêt national (1713-1795). Les cosmopolitiques du droit des gens, Paris, Kimé, 1998.

82. Voir A.N., BB18, dossier 3308, 57 pièces. État des poursuites judiciaires dans le ressort de la cour royale d'Angers, sur les circonstances du duel, le 18 février 1818, entre Rigomer Bazin et Fortuné Dessauguion.

83. Un mois après sa mort, une brochure réclame l'ouverture d'une souscription ouverte aux patriotes de la Sarthe, pour la construction d'un mausolée. L'auteur invite à poursuivre le travail commencé et perceptible dans le département, où déjà des écoles gratuites d'enseignement mutuel ont ouvert, et propose de soutenir le « gouvernement de Louis XVIII qui marche franchement avec l'esprit du siècle ", dans Denis-Claude BARBIER, À la Mémoire de Rigomer Bazin... Pierre chez Ariste, chez l'auteur, rue de la Comédie, n 10, à Angers, février 1818, B.N.F. Ln27 22417.

84.Cf. François DORNIC (dir.) Histoire du Mans, Toulouse, Privat, 1975. Les chapitres sur la période révolutionnaire et le premier XIX ${ }^{\mathrm{e}}$ siècle, sont de Paul BOIS et Marc AUFFRET, qui précisent que, jusque sous le Second Empire, toutes les élections municipales, sauf celle de 1835, vont assurer aux républicains de larges victoires et conférer au Mans le surnom d'« impôt républicain dans la France monarchiste », pp.214-246.

\section{RÉSUMÉS}

Le parcours de Rigomer Bazin (1769-1818), permet de mieux saisir les diverses attitudes que les républicains démocrates, fidèles au projet de construction d'une démocratie représentative, doivent adopter durant le Directoire, le Consulat, l'Empire, et la Restauration, face aux divers appareils de censure auxquels ils sont confrontés.

La vie politique de la Restauration jusqu'en 1818, permet au journaliste constitutionnel du Mans, de penser les conditions d'adaptation de la Charte aux idées républicaines. Au cœur de ce projet d'émancipation de l'opinion publique, se trouvent le désir de fondation d'une citoyenneté active et la volonté de réalisation des conditions objectives d'un jeu politique, respectueux des droits de l'opposition par l'alternance des partis au pouvoir. L'histoire et l'éducation doivent constituer les fondements d'un civisme nouveau. Une république confédérale européenne représenterait l'étape suivante de l'union d'un continent pacifié, autour d'un programme politique partagé et discuté en son parlement. 
Rigomer Bazin and the Restoration Making Sense of the Republic under the Monarchy. The course travelled by Rigomer Bazin (1769-1818) sheds light on the attitudes and constraints which democratic republicans were forced to contend with in their struggle to build a representative democracy during the Directory, Consulate, Empire and Restoration, in the face of multiple forms of censorship.

The political life of the Restoration till 1818 gave the constitutional journalist from Le Mans the opportunity to mull over ways of adapting the Charter to republican ideas. At the heart of this attempt to emancipate public opinion, we find the desire to forge active citizenship and achieve the objective conditions of fair play in politics through respect for the rights of the opposition and allowing each party their turn in power. History and education would become the foundations of modern civics. A federal European Republic would be the next stage of political union on a continent free from strife, through a shared political programme debated in her parliament.

INDEX

Mots-clés : Bazin, Restauration, constitution, opinion publique, opposition républicaine

\section{AUTEUR}

\section{PIERRE SERNA}

Institut d'Histoire de la Révolution française, Université Paris I Panthéon-Sorbonne 51, rue Sacco et Vanzetti, 94800 - Villejuif

vpserna@worldnet.fr 